\title{
Traditional Chinese medicine to treat COVID-19: the importance of evidence-based research
}

\author{
Jinjing Wang, Fanghua Qi* \\ Department of Traditional Chinese Medicine, Shandong Provincial Hospital Affiliated to Shandong First Medical University, Ji'nan, Shandong, China.
}

\begin{abstract}
SUMMARY Coronavirus disease 2019 (COVID-19) broke out in 2019 and spread rapidly around the world, causing a global pandemic. Traditional Chinese medicine has a history of more than 2,000 years in the prevention and treatment of epidemics and plagues. In guidelines on fighting COVID-19, the National Health Commission (NHC) has recommended some traditional Chinese medicines (TCM), including Jinhua Qinggan granules, Lianhua Qingwen capsules, XueBijing injections, a Qingfei Paidu decoction, a Huashi Baidu decoction, and a Xuanfei Baidu decoction. Based on current results, TCM has displayed some efficacy in combating COVID-19. However, TCM faces many challenges in terms of being recognized around the world. Therefore, evidence-based research is crucial to the development of TCM.
\end{abstract}

Keywords traditional Chinese medicine, COVID-19, evidence-based research

Coronavirus disease 2019 (COVID-19) broke out in 2019 and spread rapidly around the world, causing a global pandemic. As of June 30, 2020, more than 10 million confirmed cases of COVID-19 have been documented globally. Unfortunately, there is still no specific vaccine or drug approved for the treatment of COVID-19 (1). Traditional Chinese medicine (TCM) has a history of more than 2,000 years in the prevention and treatment of epidemics and plagues. In light of its experience treating SARS and H1N1 influenza, the National Health Commission (NHC) has recommended TCM as a strategy for COVID-19 treatment.

As of March 4, 2020, the NHC has issued 7 versions of its guidelines for diagnosis and treatment of COVID-19. TCM to prevent and control COVID-19 has been included since the $3 \mathrm{rd}$ version. Based on the current results, TCM has displayed some efficacy in combating COVID-19 (2). The public is well aware of the clinical efficacy and safety of TCM for treatment of COVID-19, and numerous studies in that respect have been conducted. As of June 20, 2020, more than 1,000 articles are available on PubMed when searching with the keywords "COVID-19" and "Chinese medicine", and about 150 clinical trials on TCM for treatment of COVID-19 (20.0\% of the total trials) have been registered on the Chinese Clinical Trial Registry (3).

According to data presented by the National Administration of Traditional Chinese Medicine on March 23, 2020, 74,187 patients diagnosed with
COVID-19 (accounting for $91.5 \%$ of the sample) have used TCM in China, and the total efficacy of TCM is as high as $90 \%$ based on observations (4). Moreover, "three patented Chinese medicines and three TCM prescriptions" have proven to be efficacious in treating COVID-19. The three patented Chinese medicines are Jinhua Qinggan granules, Lianhua Qingwen capsules, and XueBijing injections, and the the three TCM prescriptions are a Qingfei Paidu decoction, a Huashi Baidu decoction, and a Xuanfei Baidu decoction (5).

Jinhua Qinggan granules have been found to have equivalent efficacy to that of oseltamivir in combating H1N1 influenza (6). Patients with COVID-19 taking Jinhua Qinggan granules recovered faster than patients who did not take the granules, testing negative for coronavirus more than two days sooner (7). Therapeutic efficacy was significantly higher in patients with COVID-19 taking Lianhua Qingwen capsules ( $n=$ $147)$ and Arbidol (umifenovir) $(n=148)$ than that in patients taking Arbidol alone (80.95\% vs. $64.86 \%, p$ $=0.002$ ), and the conversion rate to severe disease in patients taking the capsules was significantly lower than that in patients taking Arbidol alone (14.29\% vs. $23.65 \%, p=0.040$ ) (8). A Qingfei Paidu decoction has also displayed noteworthy efficacy in treating COVID-19. Two hundred and fourteen patients with confirmed COVID-19 in four provinces took 3 courses of the decoction (9). More than $60 \%$ of those patients displayed obvious improvement in symptoms 
and computed tomography (CT) findings, and the remaining $30 \%$ were in stable condition without disease progression.

Although TCM has played an important role in fighting COVID-19 in China, it faces many challenges in terms of being recognized around the world. For example, the journal "Nature" not long ago published an article on its website questioning traditional Chinese medicine; David Cyranoski thought it was dangerous to support therapies that have yet to be proven safe and efficacious (10). Therefore, quality clinical trials and basic research must be conducted in order to advance TCM.

In comparison to the process of treatments being developed "from the laboratory to the clinic" in Western medicine, TCM has first found to be effective in its long history of clinical use, following an opposite process "from the clinic to the laboratory". The gradual development of TCM is based on the constant accumulation and summarization of experience in clinical practice. With the development of evidence-based medicine, the rapid transition of and transformation from experience-based medicine to evidence-based medicine have become crucial to the development of TCM. Therefore, a series of prospective cohort studies or randomized controlled trials (RCTs) should be conducted to evaluate the efficacy of TCMs, yielding reliable evidence for use of TCM to prevent or treat COVID-19 or similar emerging respiratory infectious diseases in the future (11). Moreover, welldesigned in vitro cell experiments and in vivo animal studies need to be conducted and multiple modern technologies, including molecular biology, proteomics, metabolomics, genomics, and network pharmacology, should be used to confirm the ingredients, targets, and mechanisms of TCM (12). In addition, researchers should look into the safety of TCM (13).

In conclusion, more solid scientific grounds are needed if TCM is to play a bigger role on the world stage, and TCM still has a long way to go. With hope, further research will study the standardization, evaluate the safety, and explore the mechanism of action of TCM in the treatment of COVID-19 and other diseases. TCM and Western medicine should be able to complement one another and improve health worldwide.

\section{References}

1. World Health Organization. Q\&A on coronaviruses. https://www.who.int/emergencies/diseases/novelcoronavirus-2019/question-and-answers-hub/q-a-detail/ q-a-coronaviruses (accessed June 18, 2018).

2. National Health Commission of the People's Republic of China. Diagnosis and treatment of pneumonia caused by the 2019 new coronavirus (2019-nCoV). http://www.nhc. gov.cn/wjw/xinx/xinxi.shtml (accessed June 18, 2018).

3. New Coronavirus Pneumonia Clinical Research Index. Chinese Clinical Trial Registry. http://www.chictrorg.cn/ index.aspx (accessed June 20, 2020).

4. National Administration of Traditional Chinese Medicine. The State Council Office holds a press conference on the important role and efficacy of traditional Chinese medicines in preventing and treating COVID-19. http:// www.scio.gov.cn/xwfbh/xwbfbh/wqfbh/42311/42768/index. htm (accessed June 20, 2020).

5. National Administration of Traditional Chinese Medicine. Practitioners of traditional Chinese medicine select a batch of formulations, as exemplified by "three existing TCMs and 3 new formulations", to treat COVID-19. http://www. satcm.gov.cn/xinxifabu/meitibaodao/2020-03-23/14191. html (accessed June 20, 2020). (in Chinese)

6. Wang C, Cao B, Liu QQ, et al. Oseltamivir compared with the Chinese traditional therapy Maxingshigan-Yinqiaosan in the treatment of H1N1 influenza: a randomized trial. Ann Intern Med. 2011; 155:217-225.

7. Ren JL, Zhang AH, Wang XJ. Traditional Chinese medicine for COVID-19 treatment. Pharmacol Res. 2020; 155:104743.

8. Yu L, LI YZ, Wang S, Wang Y. Observation of the efficacy of Lian-hua Qing-wen Granules combined with Arbidol in the treatment of mild COVID-19. Chinese Pharmaceutical Journal. http://kns.cnki.net/kcms/detail/11.2162. R.20200422.1429.002.html (accessed June 20, 2022).(in Chinese)

9. Cao P, Wu S, Wu T, Deng Y, Zhang Q, Wang K, Zhang Y. The important role of polysaccharides from a traditional Chinese medicine-Lung Cleansing and Detoxifying Decoction against the COVID-19 pandemic. Carbohydr Polym. 2020; 240:116346.

10. David Cyranoski. China is promoting coronavirus treatments based on unproven traditional medicines. Nature. https://www.nature.com/articles/d41586-02001284-x (accessed June 20, 2022).

11. Liu B, Zhang Y, Hu J, He L, Zhou X. Thinking and practice of accelerating transformation of traditional Chinese medicine from experience medicine to evidencebased medicine. Front Med. 2011; 5:163-170.

12. Huang YF, Bai C, He F, Xie Y, Zhou H. Review on the potential action mechanisms of Chinese medicines in treating Coronavirus Disease 2019 (COVID-19). Pharmacol Res. 2020; 158:104939.

13. Cai P, Qiu H, Qi F, Zhang X. The toxicity and safety of traditional Chinese medicines: Please treat with rationality. Biosci Trends. 2019; 13:367-373.

Received June 2, 2020; Revised June 27, 2020; Accepted June 29, 2020.

*Address correspondence to:

Fanghua Qi, Department of Traditional Chinese Medicine, Shandong Provincial Hospital affiliated to Shandong First Medical University, No.324, Jingwuweiqi Road, Ji'nan 250021, Shandong, China.

E-mail: qifanhgua2006@126.com 